\title{
Ground States of the Falicov-Kimball Model Extended by Nonlocal Coulomb Interactions
}

\author{
H. ČenČARIKOvÁ, P. FARKAŠOvskÝ AND M. ŽondA \\ Institute of Experimental Physics, Slovak Academy of Sciences \\ Watsonova 47, 04101 Košice
}

\begin{abstract}
The small-cluster exact-diagonalizations are used to study the ground states of the Falicov-Kimball model extended by nonlocal Coulomb interactions (the nearest-neighbour interaction $U_{\mathrm{nn}}$ and the correlated hopping $t^{\prime}$ ). It is shown that the ground-state phase diagrams found for the conventional Falicov-Kimball model are strongly changed when the nonlocal interactions are added. This is illustrated for two selected values of the on-site Coulomb interaction $(U)$ that represent typical behaviours of the model in the intermediate and strong coupling limit. A number of remarkable results are found. (i) The phase separation takes place for a wide range of $U_{\mathrm{nn}}$ and $t^{\prime}$ in both interaction limits. (ii) New types of inhomogeneous charge ordering are observed for nonzero $U_{\mathrm{nn}}$ and $t^{\prime}$. (iii) Depending on the values of $U_{\mathrm{nn}}$ and $t^{\prime}$, the model is able to describe both the continuous as well as discontinuous changes of the $f$-electron occupation number.
\end{abstract}

PACS numbers: 75.10.Lp, 71.27.+a, 71.28.+d

\section{Introduction}

Since its introduction in 1969, the Falicov-Kimball model (FKM) has become an important standard model for the description of correlated fermions on a lattice. The model was originally proposed to describe the metal-insulator transitions in the rare-earth and transition-metal compounds [1]. Later it has been used to study such phenomena as alloy formation, mixed valence and electronic ferroelectricity [2]. Recent theoretical studies of the spinless version of FKM showed that although this model is relatively simple, it can yield the correct physics for describing the ground states of rare-earth and transition-metal compounds [2]. On the other hand, it should be noted that the conventional FKM neglects all nonlocal interactions and thus it is questionable whether above mentioned results persist also in more realistic situations when nonlocal interactions will be turned on. In this paper we examine effects of the nearest-neighbour Coulomb interaction $U_{\mathrm{nn}}$ 
between $f$ and $d$ electrons in a combination with correlated hopping term $t^{\prime}$, that are of the same order. The fundamental question that we would like to answer here is whether or not these nonlocal interactions can describe discontinuous valence transitions from an integer/intermediate valence state with $n_{f}>0.5$ to an intermediate valence state with $n_{f}<0.5$, as observed experimentally in some rare-earth compounds, e.g., SmS. Thus our starting Hamiltonian has the form

$$
\begin{aligned}
H= & t \sum_{\langle i, j\rangle} d_{i}^{+} d_{j}+t^{\prime} \sum_{\langle i, j\rangle}\left(f_{i}^{+} f_{i}+f_{j}^{+} f_{j}\right) d_{i}^{+} d_{j}+U \sum_{i} f_{i}^{+} f_{i} d_{i}^{+} d_{i} \\
& +U_{\mathrm{nn}} \sum_{\langle i, j\rangle} f_{j}^{+} f_{j} d_{i}^{+} d_{i},
\end{aligned}
$$

where $f_{i}^{+}\left(f_{i}\right)$ are the creation (annihilation) operators for an electron in the localised state at lattice sites $i$ and $d_{i}^{+}\left(d_{i}\right)$ are the creation (annihilation) operators of the itinerant spinless electrons in the $d$-band Wannier state at site $i$. The first term is the kinetic energy corresponding to quantum mechanical hopping of $d$ electrons between the nearest-neighbour sites $i$ and $j$. The second term is the correlated hopping term. The influence of this term on the ground states has been studied recently in [3]. The third term is the on-site Coulomb interaction between the $d$-band electrons with density $n_{d}=\frac{1}{L} \sum_{i} d_{i}^{+} d_{i}$ and $f$ electrons with density $n_{f}=\frac{1}{L} \sum_{i} f_{i}^{+} f_{i}$, where $L$ is the number of lattice sites (in this paper we consider the half-filled band case $n_{f}+n_{d}=1$ ). Finally, the last term is just the nonlocal Coulomb interaction between $d$ electron on site $i$ and $f$ electrons on the nearest neighbour sites (the similar subject, the influence of nonlocal $f-f$ interaction on the ground states of the FKM has been studied in [4]). Since the $f$-electron density operator $f_{i}^{+} f_{i}$ commutes with the Hamiltonian, $f_{i}^{+} f_{i}$ can be replaced by the classical variable $w_{i}=0,1$ and then the exact-diagonalization technique, or well-controlled numerical method [5] can be used directly to study the ground states of the Hamiltonian.

\section{Results and discussion}

To study the influence of $U_{\mathrm{nn}}$ and $t^{\prime}$ on the ground-state properties of the FKM in 1D we have performed an exhaustive numerical study of the model for $U=2$ and $U=8$ and for a wide range of $U_{\mathrm{nn}}$ as well as $t^{\prime}$ on different clusters up to $L=24$. Since both nonlocal interaction terms are of the same order, we consider here only the case $U_{\mathrm{nn}}=t^{\prime}$. To separate contributions from $U_{\mathrm{nn}}$ and $t^{\prime}$, we have started our study with the case $t^{\prime}=0$. The obtained phase diagrams in $n_{f}-u_{\mathrm{nn}}$ plane $\left(u_{\mathrm{nn}}=U_{\mathrm{nn}} / U\right)$ are displayed in Fig. 1.

Our results clearly demonstrate that already relatively small changes of $u_{\mathrm{nn}}$ can produce large changes in the ground-state $f$-electron distributions. Indeed, for $U$ large we have found that already values of $U_{\mathrm{nn}}$ around 60 times smaller than $U$ $\left(u_{\mathrm{nn}}^{\mathrm{c}}=0.015\right)$ are able to destroy fully the most homogeneous $\left(w_{\mathrm{MH}}\right)$ distributions of $f$ electrons (that are the ground states of the conventional FKM in the strong 

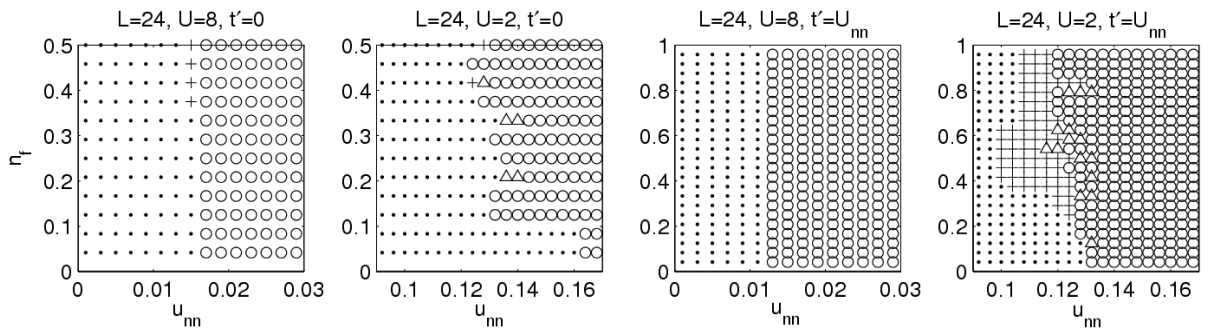

Fig. 1. Ground-state phase diagrams of the spinless FKM extended by $U_{\mathrm{nn}}$ and $t^{\prime}$ for $U=8$ and $U=2$ (for $L=24$ ). (.) $w_{\mathrm{MH}},(\circ) w_{\mathrm{s}},(\Delta) w_{\mathrm{Ps}}$, and $(+) w_{\mathrm{h}}$ distributions. The exact diagonalisation results.

coupling limit for all $n_{f}$ ). It is interesting that only two configuration types are stabilised above $u_{\mathrm{nn}}^{\mathrm{c}}$. The first configuration type $\left(w_{\mathrm{h}}\right)$ is formed by the homogeneous distribution of 1100 and 10 clusters (e.g., 110010011001001100100100 for $N_{f}=10,110011001100110011001100$ for $\left.N_{f}=12\right)$. The second type of configurations determined in the phase diagram (for $U=8$ ) are the segregated $\left(w_{\mathrm{s}}\right)$ configurations (all $f$ electrons clump together), that are preferred as a ground state for all $n_{f}$ started at $u_{\mathrm{nn}}=0.016$. The same behaviour, and namely, by $u_{\mathrm{nn}}$ induced transitions from regular to phase segregated distributions, holds also for smaller values of $U(U=2)$. In this region the ground-state phase diagram is formed by the $w_{\mathrm{MH}}$ configurations $(\cdot)$, the $w_{\mathrm{s}}$ configurations (o), the weakly perturbed segregated $\left(w_{\mathrm{Ps}}\right)$ configurations $(\triangle)$ (e.g., 111100000010000000000000 , $111111110010010000000000)$ and $w_{\mathrm{h}}$ distributions $(+)$.

Let us now examine the case when both $u_{\mathrm{nn}}$ and $t^{\prime}$ are switched on. In Fig. 1 we present the ground-state phase diagrams obtained for $U=8$ and $U=2$. One can see that the strong coupling phase diagram $(U=8)$ is practically identical to the case of $t^{\prime}=0$. For both cases the basic structure of the phase diagrams is formed by two configuration types $\left(w_{\mathrm{MH}}\right.$ and $\left.w_{\mathrm{s}}\right)$ and only one difference is that the stability region of $w_{\mathrm{s}}$ configurations shifts to lower values of $u_{\mathrm{nn}}$. Qualitatively the same picture is observed also for smaller values of $U(U=2)$. Again the segregated region is stabilised for nonzero values of correlated hopping, and in addition the stability region of $w_{\mathrm{h}}$ configurations considerably increases. Having the complete set of ground-state configurations we tried to construct the comprehensive picture of valence transitions within the FKM extended by nonlocal interactions. Since relatively large finite-size effects have been observed on clusters up to $L=24$ sites, we have used a well-controlled numerical method to construct the $n_{f}\left(E_{f}\right)$ behaviour. Representative examples of $n_{f}\left(E_{f}\right)$ behaviour obtained for various combinations of $u_{\mathrm{nn}}$ and $t^{\prime}$ are shown in Fig. 2. For zero values of $u_{\mathrm{nn}}$ and $t^{\prime}$ the valence transition has the typical staircase structure (the conventional FKM). By switching on $u_{\mathrm{nn}}$ and $t^{\prime}$ it is possible to induce the continuous valence transition $\left(u_{\mathrm{nn}} \neq 0\right)$, or a sequence of several discontinuous and continuous transitions $\left(t^{\prime} \neq\right.$ $0)$. From the theoretical and experimental point of view the most interesting 

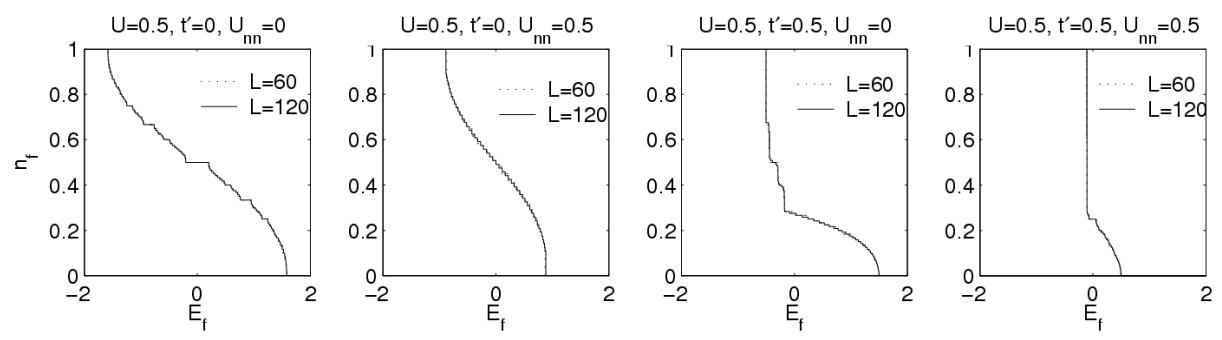

Fig. 2. Dependence of the $f$-electron occupation number $n_{f}$ on the $f$-level position $E_{f}$ for $L=60$ and 120 at $U=0.5$, depicted for different values of $U_{\mathrm{nn}}$ and $t^{\prime}$.

valence changes are induced when both $u_{\mathrm{nn}}$ and $t^{\prime}$ are switched on simultaneously. In this case there is only one discontinuous valence transition from an integer valence state $n_{f}=1$ to an intermediate valence state $n_{f}<0.5$ and above the transition point the occupation number $n_{f}$ changes continuously.

This picture is in a nice correspondence with experimental data obtained for the mixed valence compound $\mathrm{SmS}$ [6]. Thus we can conclude that nonlocal interactions $\left(u_{\mathrm{nn}}\right.$ and $\left.t^{\prime}\right)$ play the crucial role in description of the ground-state properties of the spinless FKM and they should not be neglected in the correct description of real materials with correlated electrons.

\section{Acknowledgments}

This work was supported by Slovak Grant Agency VEGA under grant No. 2/7057/27 and Slovak Research and Development Agency (APVV) under grant LPP-0047-06. H.C. acknowledges support of Stefan Schwartz Foundation.

\section{References}

[1] L.M. Falicov, J.C. Kimball, Phys. Rev. Lett. 22, 997 (1969).

[2] J. Freericks, V. Zlatic, Rev. Mod. Phys. 75, 1333 (2003).

[3] J. Wojtkiewicz, R. Lemanski, Phys. Rev. B 64, 233103 (2001); H. Čenčariková, P. Farkašovský, Int. J. Mod. Phys. B 18, 357 (2004).

[4] Z. Gajek, R. Lemanski, J. Magn. Magn. Mater. 272, E691 (2004).

[5] P. Farkašovský, Phys. Rev. B 20, 1507 (2095); Eur. Phys. J. B 20, 209 (2001).

[6] J. Röhler, Handbook on the Physics and Chemistry of Rare Earths, North Holland, Elsevier, 1987, p. 510. 